\title{
Incident Report: Between the Shadows of Obligation and Formality
}

\author{
Savitri Citra Budi ${ }^{1,2 \star}$, Sunartini Hapsara ${ }^{3}$, Fatwa Sari Tetra ${ }^{4}$, Lutfan Lazuardi ${ }^{5}$ \\ ${ }^{1}$ Doctoral Program, Faculty of Medicine, Public Health and Nursing, Universitas Gadjah Mada, Yogyakarta, Indonesia; \\ ${ }^{2}$ Department of Health Information and Services, Vocational School, Universitas Gadjah Mada, Yogyakarta, Indonesia; \\ ${ }^{3}$ Department of Pediatrics, Faculty of Medicine, Public Health and Nursing, Universitas Gadjah Mada, Yogyakarta, Indonesia; \\ ${ }^{4}$ Depatment of Health Behavior, Environmental, and Social Medicine, Faculty of Medicine, Public Health and Nursing, \\ Universitas Gadjah Mada, Yogyakarta, Indonesia; ${ }^{5}$ Department of Health Policy and Management, Faculty of Medicine, Public \\ Health and Nursing, Universitas Gadjah Mada, Yogyakarta, Indonesia
}

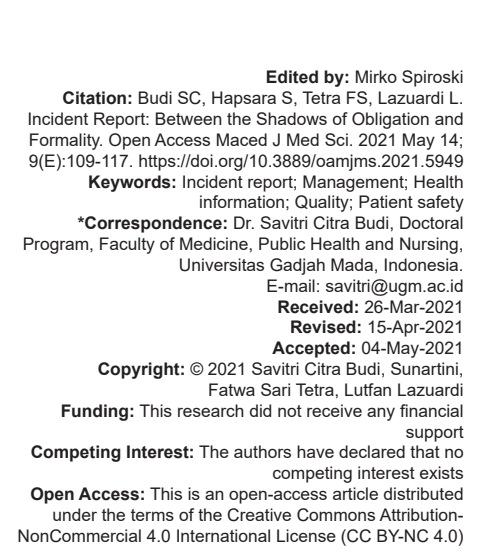

Introduction

As a place that provides health services, hospitals have a complex and high-risk system so that incidents can sometimes occur in the service process. These incidents can cause negative outcomes related to patient safety when they are receiving services at the hospital. When a pandemic happens, more potential injuries can occur with patients or health workers in hospitals, including the risk of contracting coronavirus disease-19.

Under any circumstances, the hospital must safely provide services to patients. The concept of patient safety must be applied in the patient's interest, not just because of the hospital's formality in carrying out their policy rules. Patient safety is a shared commitment to patients, families, health practitioners, staff, and health-care associations [1]. The hospital staff is expected to implement the concept of patient safety, which means that the hospital creates a safer system for patients to receive services, including risk assessment, risk identification, incident reporting and analysis, providing opportunities for those involved to learn from incidents, and follow-up solutions to minimize further similar incidents [2].

Incident reports are the primary source of data for the quality analysis process and an important resource to improve patient safety. Incident reports are used as materials for learning and for the analysis processes to prevent repeat occurrences [3]. Incident reporting involves an important issue to report dangerous situations or events. Accordingly, the World Health Organization (WHO) has issued a statement that it is mandatory for hospitals to report incidents to improve the reporting process and learning system [4]. This WHO policy impacts the prudence and accuracy of officers in providing services to reduce incidents. The absence of an event can reduce costs incurred by the hospital. The hospital policy regarding incident reporting provides an overview of the efforts to improve the quality and patient safety that the hospital has made.

A report from the World Alliance for Patient Safety: Forward program 2008-2009 stated that 
unexpected incidents ranged from 3.2 to $16.6 \%$ in the United States, United Kingdom, Denmark, and Australia [5]. The National Patient Safety Agency reported 1,895,834 patient incidents in the UK from October 2016 to September 2017 [6]. Furthermore, in their research, Panesar et al. in 2017 explained that in 26 low- and middle-income countries, the frequency of unexpected events was around $8 \%$, with $83 \%$ being included in the criteria for preventable incidents [7].

Hospitals that report incidents continuously indicate that they have adopted an incident reporting culture. Therefore, hospital managers must continuously monitor by reviewing incident reports. Furthermore, the Joint Commission International (JCl) in 2014 explained that implementing an incident reporting culture is done to ensure service quality. All staff in the hospital have an essential role in implementing an incident reporting culture. Clinicians can report incident reports, while nurses and other service providers can serve as an audience effort through local, regional, and national level reporting systems and be a solution to improve patient safety [4]. Manager support for staff to report is needed so that the reported incident can be used as a learning process to prevent any repeat of the incident [8].

Sources of incident report data can come from outside parties, such as patients or families. Patients or families can report incidents through customer complaint reports, but this facility is not widely used [4]. Hospitals need to do outreach to the broader community so that patients or families can take advantage of this facility. In other words, the reporting culture in the hospital requires patient or family participation.

The incident reporting process starts from the discovery of the incident. Then, the officer creates a report that is immediately followed-up and submitted to the quality and patient safety team for the incident management process. There are two options with alternative actions at the time of the incident: (1) Actual event and report, or (2) fix it and forget [9]. Staff reluctance to report incidents is due to limited staff knowledge of incident conditions and a reasonably complex reporting system.

Research related to incident reporting is very problematic because both the high and low numbers of reported incidents directly affect service quality. For example, if an incident occurs and the officer does not report it, it remains unknown as an incident and may contribute to more adverse events in the future. As a result, the medical services to patients will continue without any learning process. Incident reporting culture is closely related to the moral responsibility of healthcare staff to report incidents. Although incidents may occur, this effort can reduce patient damage, even if the goal is always zero [1].

The Indonesian Government has implemented the international regulations endorsed by the WHO related to the implementation of incident reporting; this is evidenced by the existence of an Indonesian Government regulation that requires the implementation of patient safety in hospitals (Law Number 44 of 2009 concerning Hospitals and Indonesian Government Regulation Number 11 of 2017 concerning Patient Safety). The oversight or "shadow" of the government regulations has become the obligation of hospitals to meet the "zero" target; this occurs due to the lack of staff awareness, including hospital leaders, in viewing incident reports as the basis for patient safety.

This study aimed to assess the staff members' roles in incident reporting at the Yogyakarta Special Region Provincial Hospital, and discuss their roles and barriers in implementing this process of incident reporting. It is hoped that other researchers wishing to promote and adopt a hospital reporting culture may consider this article's findings as evidence-based support for the incident reporting policy.

\section{Methods}

\section{Study design}

This research used an exploratory qualitative research type because it investigates the roles and barriers of staff in hospitals' incident reporting process. Exploratory qualitative research gives the researcher flexibility to investigate participants' responses and uses "probes" to encourage informants to express their views [10]. The study protocol was approved by the local Medical and Health Research Ethics Committee.

\section{Informants}

The informants were hospital staff, three doctors, two recovered patients, one nurse, one pharmacist, and two computer administrators. Informants were selected by purposive convenience sampling method through the patient safety team at the hospital. The addition of informants used the recommendation method from the previous informants (snowball) [11]. The addition of informants was done to obtain a complete technical picture regarding the implementation of incident report management in the hospital because each staff member has different experiences in finding incidents and reporting them. The sample size of this study was the amount needed until data saturation was reached. The inclusion criteria for informants were staff who have worked for at least 3 years and are willing to be involved in this research. All participants gave written consent.

\section{Data collection}

The interview guide was prepared in a semistructured form and derived from the $\mathrm{JCI}$ [12]. Standards 
were translated into Indonesian (National Language of the Republic of Indonesia) and adjusted to the Ministry of Health's Republic of Indonesia regulations. Semi-structured interviews were conducted to explore officers' views on their role in incident reporting in hospitals. The interview guide was tested on experts to get input and determine feasibility. The interview guide contains information on the consent to be a respondent, the respondent's characteristics, the reporting implementation process, a feedback system, and the development of an efficient reporting system. Interviews were conducted in open and focused conversations with sufficient investigation to obtain in-depth ideas/ ideas regarding the incident reporting implementation. The interviews were audio-recorded, transcribed word by word, and analyzed systematically using the Atlas.ti application.

Further data collection was conducted by observation and documentation study. Observation data collection was used to obtain data on the continuity of the hospital's incident reporting process. Researchers conducted a documentation study on the incident report archive in 2016-2018 and a current guide to implementing the hospital's incident reporting process.

\section{Data analysis}

Researchers conducted data analysis started from data reduction, data presentation, and concluding [13]. Data reduction was done by summarizing, coding, and tracing the interview results' themes related to incident reporting management. The data analysis process was conducted simultaneously using the Atlas.ti 8 application. Data presentation was done by compiling data reduction results in the form of text and charts to conclude the analysis results. Efforts to conclude were made by considering the results of data analysis followed by verifying the research data. To check the validity of the research data, this study used a triangulation of methods, which is a qualitative research strategy to test data validity through the convergence of information from various sources [14].

\section{Results}

Results showed that informants develop a patient safety culture as an alternative effort to improve patient safety quality in the hospital. Incident reports are a source of monitoring data for the implementation of safety culture. This study used qualitative data analysis to explore the application of incident report management in hospitals.

Figure 1 presents a network image of the results of a qualitative analysis related to the management of incident reports at a regional hospital in Yogyakarta,

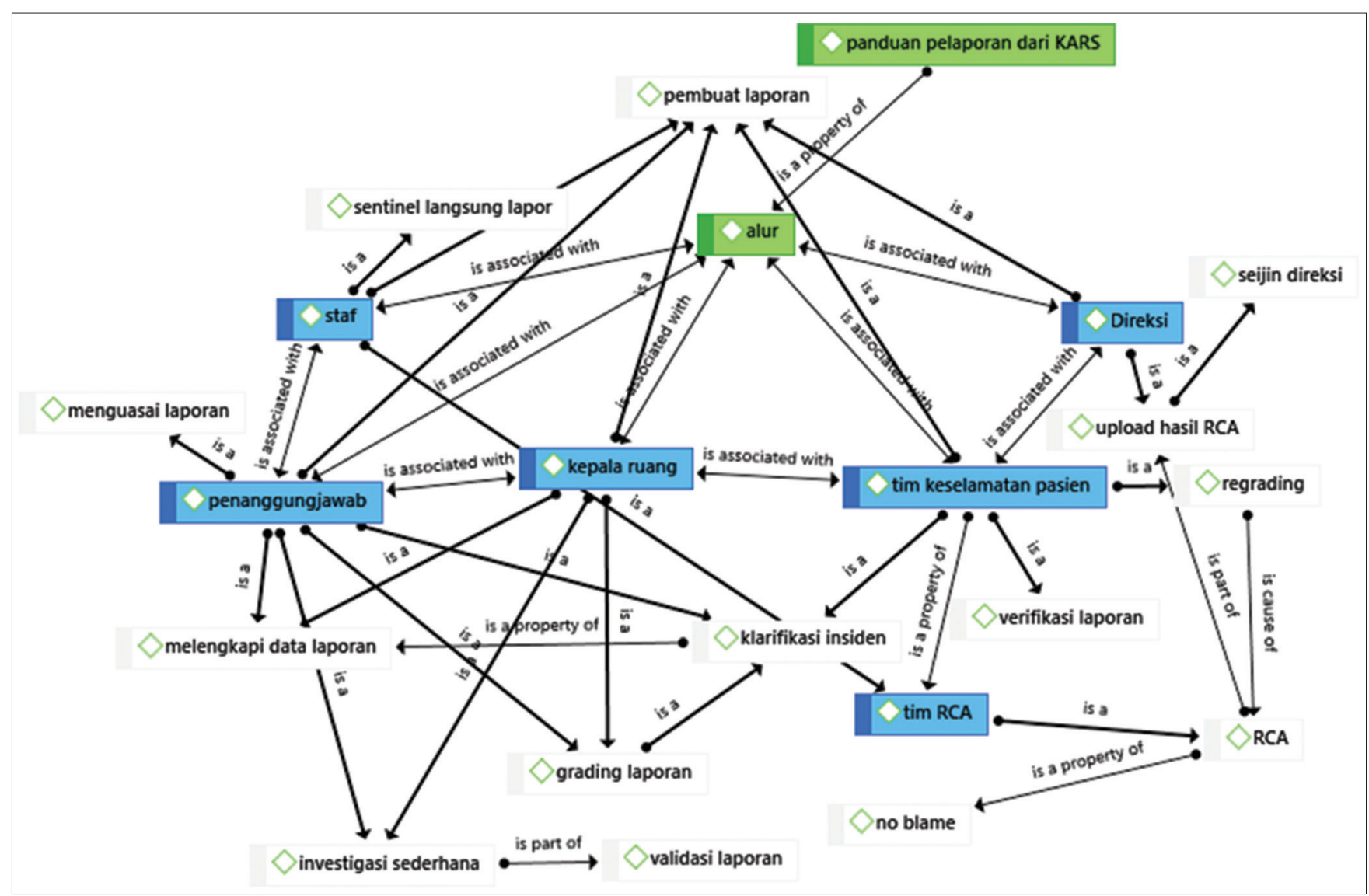

Figure 1: Management of incident reports 
Indonesia. The qualitative data analysis results found that the most prominent discussion was related to the incident reporting guide, mainly discussing the reporting flow (Figure 1 in green). Figure 1 illustrates the role of staff in the hospital in incident reporting, consisting of all staff, the report coordinator in each room, the head of the room, the patient safety team, the ad hoc team for Root Cause Analysis (RCA), and the hospital manager (indicated by blue). The findings on each staff member's roles are depicted in the box associated with the staff position (shown in Figure 1 with a white box).

Each staff member has an important role in creating an incident reporting culture. Regardless of their respective primary duties, all staff should report incidents or adverse events that could lead to patient safety incidents during treatment. Staff understanding of their role in the incident reporting system reflects their appreciation for their professional duties and responsibilities. Each staff's role in managing incident reports in the hospital is described below, including the role of staff, the role of coordinator, the role of the head of the room, the role of the patient safety team, and the role of the hospital director.

\section{Role of reporting staff}

Role of staff in incident reporting is seen as "whistleblowers." Staff is expected to report events that occur or conditions that could potentially cause injury to the patient during treatment. The staff creates a report by filling out a form submitted manually to the person in charge of the report in each room. Before reporting, the health worker provides treatment to the patient, as quoted below:

"When an incident occurs, we first report it to the on-duty doctor, and then we treat it and make observations. After we finish reporting it again to the doctor on duty, make a written report using a form, and submit a report to the person responsible for the report" (ID: 24, ES, 18 years).

No staff member is permitted to keep duplicate incident reports to keep the report confidential. In some cases, staff sometimes only gave incident reports verbally to the person in charge of the reports or the head of the room due to difficulties in accessing the manual report form, as shared in the following interview excerpt:

"Other staff does not know where the report form is. As far as I know, the report form is in the locker of the person in charge of reports" (ID: 17, WI, 14 years).

Staff understanding toward the importance of reporting incidents is still lacking. There are still many staff who never report an incident because they feel they have never experienced or seen an incident, as mentioned in the following interview:
"It just so happens that I have never encountered an incident, so I have never reported it" (ID: 23, EK, 21 years).

Staff responsibility for patient safety is also felt to be lacking because of the fear of punishment. Staff is sometimes afraid to report incidents because management may mistake them as the wrong-doer and punish them, as quoted below:

"Sometimes there are obstacles because employees do not report because they are afraid that the management will mistake them and punish them, but it depends on each person. If the intention is good, they will report it" (ID: 17, WI, 14 years old).

On the other hand, the hospital guarantees a policy not to punish staff and make incident reports a learning process.

\section{reporting \\ Role of the coordinator in incident}

The reporting coordinator and head of room receive a letter of assignment for incident report management from the director. The incident reporting coordinator is the person in charge of incident reporting whose job it is to double-check, complete the chronology of events written by the unit reporting staff, and write down the incident report register before the report is submitted to the patient safety team.

"I wrote the incident in the register in the room" (ID: 18, TF, 6 years).

The nurse is expected to write all actions on the patient's medical record, including actions resulting from the incident. However, in practice, not all actions are written in the patient's medical record, such as in the following interview:

"For example, in a patient with an incidence of falling out of bed, the nurse writes on the nursing assessment form the nurse provides initial action and puts in bed restraints" (ID: 16, PJ, 28 years).

The person in charge of incident reporting is also in charge of coordinating with the head of the room for the incident management process. The Head of the Room and the person in charge of incident reporting will conduct a simple investigation into the incident report with a yellow and green assessment. The person in charge of reporting incidents regularly coordinates with the patient safety team at the hospital.

The results of this study found that the disciplinary attitude of the incident reporting coordinator was still lacking. The coordinator did not immediately make a written report when he received incident information from other staff. The incident report coordinator did not thoroughly fulfill their responsibility to provide report forms in the room. Staff complained about the difficulty of finding the incident report form. 


\section{Role of head of room in incident reporting}

The head of the room has the role of motivating staff to report conditions that have the potential to cause incidents and activities that unintentionally cause harmful incidents to patients, as discussed in the results of interviews with informants:

"We emphasize the honesty of our staff to report the incidents that occurred. Besides, we always remind staff to make a report immediately. We motivate staff that reporting things that are detrimental to patients is not something terrible, but a means to improve facilities or resource competence" (ID: 16, PJ, 28 years).

The head of the room motivates staff to report by providing an understanding of how to report incidents. Besides, the head of the room reminded the staff of the conditions that were reported as incidents. The head of the room emphasized that staff honesty is essential for implementing an incident reporting culture. The head of the room motivates staff in the room's internal meeting. This motivation can reduce the reporter's stress concerning an incident. Explaining the importance of reporting this problem, the head of the room also motivates any staff involved to immediately complete the report no later than $2 \times 24 \mathrm{~h}$ after the incident. The results of interviews with informants on this issue stated:

"There are still difficulties in filling out the report right before $2 \times 24 \mathrm{~h}$. Sometimes the officer concerned does not make it right away, so he needs to be reminded to make an incident report immediately after the incident" (ID: 16, PJ, 28 years old).

The head of the room's responsibilities in motivating staff to report is still deemed ineffective. The staff has not been motivated with complete honesty to report incidents encountered while providing services to patients. Besides, the head of the room's responsibility is to validate incident reports that have previously been checked for completeness by the person in charge of the report.

"Validation is carried out after communicating with the officer who makes the report, the person in charge of the report, and the head of the room" (ID: 16, PJ, 28 years).

"The head of the room gets the report from the person in charge of the report and validates the report by checking the chronology of the incident" (ID: 18, TF, 6 years).

Internal meetings also discuss incident reports, evaluating them to improve the quality of service to patients. Discussion of events in the internal meeting of this room aims to make the incident report a learning process so that no incident reoccurs. The head of the room ensures that there are no penalties for staff who report incidents.
The role of the patient safety team in incident reporting

The patient safety team plays a role in creating an incident reporting culture based on patient safety goals in the hospital. Monitoring the reporting process, completing, validating, and analyzing incident reports are essential for an effective feedback process.

"Sometimes the person in charge of the report and the room nurse are invited to follow-up on the incident, and this is also an incident feedback process" (ID: 18, TF, 6 years).

The hope is, by disseminating the results of the analysis at internal hospital meetings, staff will be motivated to continue reporting incidents honestly. The patient safety team performs the analysis process by grouping incidents based on patient safety objectives and reporting back incidents at the hospital level. If the re-grading results at the hospital level have a high and extreme impact, the expert team will then conduct a RCA for a set period of time. The results of the RCA report are in the form of recommendations to the director.

The dissemination of monitoring results and incident report analysis is presented in an informative table format. The patient safety team produces a report that is addressed to the board of directors. Besides, the team sends a report to the National Hospital Accreditation Committee through the incident report submission application. The patient safety team regularly conducts coordination meetings attended by the patient safety team members and the person in charge of reports in the room.

The patient safety team comes from professional representatives who have primary duties as structural officers and staff who provide patients' services. The patient safety team has obstacles in managing incident reports. The excessive workload is in addition to their primary duties, other than as a patient safety team member. Difficulty in finding time for discussion between patient safety teams occurs due to members having different work units.

\section{reporting}

\section{Role of the board of directors in incident}

The board of directors at the hospital has a role in monitoring patient safety, especially the incident report. The board of directors is also responsible for disseminating the RCA results to related units and orders to improve service.

On the other hand, the board of directors' responsibility in monitoring incident reports was still not fully implemented. Monitoring incident reports have not become the main focus in ensuring patient safety in the hospital. 


\section{Discussion}

Hospitals in Indonesia apply regional government regulations (Government Regulation Number 11 of 2017 concerning Patient Safety), especially regarding efforts to maintain service quality and patient safety. The government oversees the implementation of these regulations by monitoring incident reports and their handling efforts. All staff must report incidents and provide first aid when an incident occurs. Management reporting and improvement efforts are done based on reports from staff in the incident report format. A precise chronology helps the manager make the right decisions for improvement, motivation, and assignment efforts.

On the other hand, patient safety incident reports sometimes do not provide an accurate picture of incidents. However, these incident reports are invaluable for understanding latent events that can harm the patient [15]. The application of incident report management in the hospital shows that efforts to improve services by prioritizing patient safety have been done. Moreover, incident report management is included in the accreditation assessment criteria, and this is a motivation for the leadership to continue to implement and monitor managing this report system.

An incident is defined as an unexpected event that occurs in a patient, causing harm to the patient during hospitalization [16]. Management of incident reports at Regional Hospitals in Yogyakarta, Indonesia, is conducted by applying the Indonesian Hospital Accreditation Committee guidelines. The implementation of the incident report management flow based on the hospital's existing work culture is implemented in each staff's role in incident report management. All staff plays a role to (1) report incidents, including: (2) The person in charge of incident reports. (3) Head of the room who has the task of validating reports. (4) The patient safety team is in charge of analyzing incident reports, and (5) the board of directors is the highest policymaker in the hospital. The incident reporting process involves staff in charge of reporting incidents, checking completeness, performing data validation, and analysis. All staff have a role in identifying and minimizing any and all treatment risks for patients [17].

All staff at the Regional Hospital in Yogyakarta, Indonesia, have to make an incident report after finding an incident. The staff's incident reports must be kept confidential until the patient safety team finally analyzes and makes remedial efforts. Incident reports that have been analyzed need to be submitted to staff as a form of the feedback system.

At present, staff has barriers to reporting incidents, including fear of punishment for providing services that cause injury to patients. On the other hand, the hospital does not punish or sanction staff for the reports submitted. Another classic obstacle is related to reporting facilities such as guides and forms, which are not easily accessible by staff. One study reported that, the incident reporting process's obstacle was due to the absence of staff responsible for patient safety at the hospital. In contrast, one common cause of incidents found was due to ineffective teamwork (doctor and nurse) in providing services and patients who did not know who should be contacted when an incident occurs [18]. It must be recognized that management staff of facilities motivate staff to report incidents. Patient safety guidelines in South Africa state that health-care facilities need to develop encouraging guidelines and protocols to motivate staff to report incidents [19].

Another research mentions that the staff has an essential role in developing a culture of reporting incidents. The staff is a dimension that is closely related to patient safety. The staff dimension in patient safety is also closely related to the recruitment process, staff placement, and supervision. Apart from the staff, work environment conditions also significantly affect patient safety when providing health services in hospitals [20].

The incident reporting requires the coordinator and the head of the room, as middle-level managers to coordinate and to cross-check the incidents and complete the report chronology. An incident register is created at room level for the internal incident report analysis process. Middle-level managers should motivate staff to report incidents. Middle-level managers have a role in motivating staff to support a successful incident reporting culture. Motivation is energy that can activate, direct, and maintain behavior [21]. In this case, the role of the advanced manager, in the patient safety team, is in charge of providing feedback based on the reports sent. The staff has not felt any feedback from the patient safety team on the incident reports that have been sent. The staff did not know whether the action taken was correct, and the hospital was trying to anticipate the same incident happening again. In social cognitive theory (Bandura, 1986), environmental and personal factors and feedback provide cues that shape behavior-specific cognitive processes - for example, learning, assessment, and planning - that determine future behavioral decisions [21]. Direct feedback to health-care professionals is essential to maintain continuity of reporting [8]. The feedback process provides a positive cue for reporting by the staff and shows respect for the reporter and strengthens the reporting culture. The form of feedback to staff can be done in three ways: Giving individuals a unique number to open the system, viewing analysis results, providing email alerts to room heads, or using quick response emails and meetings [22].

The patient safety team at the Regional Hospital in Yogyakarta, Indonesia, has played the role of analyzing incident reports and addressing the impact of incidents. Presentation of information on the results of the incident report analysis is based on: (1) Patient safety goals, (2) incidence variation, and (3) the first 
officer to assist when an incident occurs (recorded in the patient's medical record). The presentation of this graphical data helps managers make decisions. If the re-grading analysis results show significant and catastrophic impacts, the patient safety team will conduct a RCA, which aims to find the most appropriate solution to prevent the same incident. This analysis process shows that transforming data into information are essential for learning organizations and effective change management [1].

The incident reporting system aims to monitor the patient safety situation, consistency in how to report incidents, and to use the reporting system for the continuous learning process, by encouraging policy changes to improve patient safety [23]. Improving patient safety is a challenge for organizations to create an adequate infrastructure [24]. Managers can provide the resources that support the sustainability of an effective and safe reporting system by: (1) Safety warnings, (2) collaboration for incident prevention, (3) patient safety survey, (4) training and implementation of an online RCA system, (5) guide to measuring patient safety, and (6) National level patient safety forums [25]. The leadership's involvement in monitoring incident reports is one of the essential policies for implementing an incident reporting culture. The hospital leadership can establish a policy that introduces risk management principles in health services to new staff members, including incident reports [17]. New staff is selected with the consideration that high morale and low work environment influence.

The director needs to focus on the development of staff as experienced in "real-life," not just as a formality for the so-called "zero" targets' achievement. Staff development has a primary focus on instilling discipline, honesty, and responsibility in patient care that prioritizes patient safety. The disciplined attitude of applying incident reports management involves staff with full awareness to fulfill their duties immediately according to their role, not because of formality or pressure from the leadership. An honest attitude in managing incident reports includes staff reporting incidents following the chronology that actually led to the incident. The application of a responsible attitude in managing incident reports is that staff can accept the consequences of an incident and can immediately resolve the incident by a learning process that is designed to identify preventive solutions and not "scapegoats" as commonly occurs in "blame cultures."

The Industrial Revolution 4.0 is an inevitable reason for health services to immediately adjust to using technology in the form of the Internet of things, big data, artificial intelligence, and Cloud computing to improve services. A shift in service from manual to computerized systems needs to be prepared. In other studies, this system adjustment poses a challenge in system development and the incidence coding classification process [26]. Adopting an electronic incident reporting system provides several benefits, such as an increase in the number of reported incidents, the timeliness of reporting within $48 \mathrm{~h}$, and an increased role for the nonnursing staff to report incidents [26].

Monitoring the application of an electronic reporting system requires evaluation for the continuity of the system. Based on the evaluation of the use of the electronic incident reporting system, it was found that the user was faster on the electronic system used. User ratings are based on the ease, accessibility, and consistency of the system for managing electronic incident reports [26]. Computerized systems provide a database of incident types and root causes that can help management identify trends, contribute to problems, and monitor incident data changes [8]. Besides, choosing an electronic system is expected to provide information to prevent the same incident from happening again, information on the cause of the problem, and map information on patient safety incident numbers.

The Board of Directors assigns a team consisting of various expertises according to the incident's conditions to conduct a RCA. In their next role, the Board of Directors assigns the head of the room to make improvements. Assignments are awarded based on recommendations from the results of the RCA process. The Board of Directors monitors the incident report management process at the hospital in a system that has been implemented. This role is a technical role played by the leadership to fulfill their duties, but promotion and motivation are needed to implement this incident reporting system into a hospital's reporting culture. The leader's role in the incident reporting system mainly consists of: (1) Promote reports as a learning process, (2) motivate staff to uphold a fair safety culture, (3) provide a transparent environment regarding patient safety, (4) provide a model for modeling professional behavior, (5) provide firm action for any behavior that is likely to destroy safety culture, and (6) provide resources and training for making remedial efforts [1]. Leaders should educate staff and hold staff accountable for their professional behavior to eliminate actions detrimental to the safety culture. The leadership can provide support by ensuring and encouraging the system's use for internal reporting incidents in the hospital.

Managerial support is needed to reduce barriers to implementing this reporting culture. These obstacles are in the form of a lack of support from middlelevel managers, lack of staff's knowledge and skills to report, and team skills in conducting incident analysis to determine the root cause of the problem [27]. Efforts to understand what is happening in the organization can be made by applying confidentiality according to the management structure's level, which manages information patterns, organizational structures, processes, and incident report transactions. All staff are expected keep reports confidential and make incident 
reports a learning process. Management of incident reports can be localized, viewed as expected, and will not be subject to external oversight. The distance factor, both physical and social, may interfere with understanding what is happening in the organization through a lack of transparency [28].

Building a reporting culture requires the active role of all staff. Staff and managers are the determinants of a successful incident reporting culture. Health services benefit from incident reporting through systems for improving quality of care and patient safety [26]. The active role of the incident reporting staff is aimed at data collection to promote internal quality improvement, performance appraisal, the need for insurance claims, and hospital assessments [29]. Incident reports are written in the free text; additionally, long descriptions can provide a detailed view of the incident [30]. Nurses' dominance as reporters resulted in the description of the chronology of incidents being more likely in the care process [8]. Furthermore, failure in the collaboration between units is the most frequently reported cause of incidents [8]. The quality of communication between departments can affect the occurrence of incidents.

Monitoring of the reporting culture is done to ensure that reporting runs continuously. There are four main components to optimizing an incident reporting system: Data input with a non-punitive culture, data collected in free text, and data analysis that can turn reports into learning processes, and a feedback system that can encourage further reporting [22]. The patient safety standard based on the JCAHO states that leaders must ensure a continuous proactive program to identify patient safety risks and reduce medical/health-care errors. These standards are defined and implemented in health services [31].

\section{Conclusions}

It is very important for hospitals to have a commitment to promote a fair and open culture in incident management. The leadership should always strive to instill a disciplined, honest, and responsible attitude in managing incident reports to staff in accordance with their roles.

Health services require all staff's active role according to their level to maintain a culture of incident reporting. The staff's role as reporting, the coordinator as the person in charge of the report, the head of the room that does the validation, the patient safety team that performs the analysis, and management as decision-makers, all support each other to improve patient safety.

Incident report information can be presented in trends to promote, analyze, evaluate, and monitor service quality and patient safety. The socialization of conditions can be reported as incidents need to be socialized regularly. The active engagement of all of these approaches can motivate staff to report honestly. In the future, it is necessary to develop a more effective incident reporting system so that officers can report quickly and effectively.

\section{Acknowledgment}

The authors would like to thank LPDP for help this research, School of Vocational Universitas Gadjah Mada, to support qualitative analysis software.

\section{References}

1. The Joint Commission. Patient Safety Systems. Vol. 1. Oakbrook Terrace, Illinois: The Joint Commission; 2019.

2. Armitage G, Moore S, Reynolds C, Laloë PA, Coulson C McEachan $\mathrm{R}$, et al. Patient-reported safety incidents as a new source of patient safety data: An exploratory comparative study in an acute hospital in England. J Health Serv Res Policy. 2018;23(1):36-43. https://doi.org/10.1177/1355819617727563 PMid:29235364

3. Budi SC, Sunartini, Lazuardi L, Dewi FS. Information systems and patient safety incident reports: A systematic review of literature and observational incident reporting system in hospitals. Int J Recent Technol Eng. 2019;8(1):807-14

4. World Health Organization. WHO Draft Guidelines for Adverse Event Reporting and Learning Systems. Vol. 16. Geneva, Switzerland: World Health Organization; 2006. p. 80

5. World Health Organization. Forward Programme 2008-2009. Geneva, Switzerland: World Health Organization; 2009.

6. NHS Improvement. NRLS National Patient Safety Incident Reports: Commentary. London: NHS Improvement; 2018.

7. Panesar SS, Carson-Stevens A, Salvilla SA, Sheikh A. Patient Safety and Healthcare Improvement at a Glance. Hoboken, New Jersey: Wiley; 2014.

8. Wagner C, Merten H, Zwaan L, Lubberding S, Timmermans D, Smits M. Unit-based incident reporting and root cause analysis: Variation at three hospital unit types. BMJ Open. 2016;6(6):11277. https://doi.org/10.1136/bmjopen-2016-011277

9. Hewitt T. Hospital-Based Views and Practices to Incident Reporting and Patient Safety: A Qualitative Comparative Study of Two Divisions; 2015.

10. Farr BC. Qualitative Research Methods: A Data Collector's Field Guide. Vol. 25. Durham, North Carolina: Family Health International; 2008.

11. Braun V, Clarke V. Snowball sampling completion IrinaMaria Dragan, Alexandru Isaic-Maniu. J Stud Soc Sci. 2013;5(2):160-77.

12. Joint Commission International. Joint Commission Internationa Accreditation Standards for Hospitals Standards-only Version. Oakbrook Terrace: Joint Commission International; 2014. p. 35 https://doi.org/10.37573/9781585284474.002

13. Matthew AM, Miles B. Qualitative Data Analysis: An Expanded 
Sourcebook; 1994. Available from: https://www.books google.co.id/books?hl=en\&lr=\&id=U4IU_-wJ5QEC\&oi=fn $\mathrm{d} \& \mathrm{pg}=\mathrm{PA} 10 \& \mathrm{dq}=$ buku+ miles + dan + huberman\&ots $=\mathrm{kFTD}$ IVY_U\&sig=F-NFGRPh58xZSOcKyhQh23WyT3Y\&redir esc $=\mathrm{y} \# \mathrm{v}=$ onepage \&q\&f=false. [Last accessed on $2021 \mathrm{Feb} 23$ ].

14. Carter N, Bryant-Lukosius D, Dicenso A, Blythe J, Neville AJ. The use of triangulation in qualitative research. Oncol Nurs Forum. 2014;41(5):545-7. https://doi.org/10.1188/14.onf.545-547 PMid:25158659

15. Ramírez E, Martín A, Villán $\mathrm{Y}$, Lorente M, Ojeda J, Moro M, Vara $\mathrm{C}$, et al. Effectiveness and limitations of an incidentreporting system analysed by local clinical safety leaders in a tertiary hospital: Prospective evaluation through realtime observations of patient safety incidents. Med (United States). 2018;97(38):e12509. https://doi.org/10.1097/ md.0000000000012509

PMid:30235764

16. Heideveld-chevalking AJ, Calsbeek H, Damen J, Gooszen H, Wolff AP. The impact of a standardised incident reporting system in the perioperative setting: A single center experience on 2,563 "near-misses" and adverse events. Patient Saf Surg. 2014;8(1):46. https://doi.org/10.1186/ preaccept-1149944930147258 PMid:25632301

17. National Health Service. Incident Reporting Policy and Procedure. United Kingdom: National Health Service; 2020

18. Wami SD, Demssie AF, Wassie MM, Ahmed AN. Patient safety culture and associated factors: A quantitative and qualitative study of healthcare workers' view in Jimma zone Hospitals, Southwest Ethiopia. BMC Health Serv Res. 2016;16:495 https://doi.org/10.1186/s12913-016-1757-z

PMid:27644960

19. Department Health Republic of South Africa. National Guideline for Patient Safety Incident Reporting and Learning in the Public Health Sector of South Africa April 2017. Pretoria, South Africa: Department Health Republic of South Africa; 2017. https://doi. org/10.1163/2213-2996_flg_com_172084

20. Danielsson M, Nilsen $\mathrm{P}$, Rutberg $\mathrm{H}$, Årestedt K. A national study of patient safety culture in hospitals in Sweden. J Patient Saf. 2019;15(4):328-33. https://doi.org/10.1097/ pts.0000000000000369 PMid:28234728

21. Holden RJ, Karsh BT. Applying a Theoretical Framework to the Research and Design of Medical Error Reporting Systems. Healthcare System Ergonomics Patient Safety Human Factor, a Bridge Between Care Cure Proceeding International Conference HEPS; 2005. p. 131-4; 2014.
22. Reed S, Arnal D, Frank O, Gomez-Arnau JI, Hansen R, LesterO, et al. National critical incident reporting systems relevant to anaesthesia: A European survey. $\mathrm{Br} \mathrm{J}$ Anaesth. 2014;112(3):546-55. https://doi.org/10.1093/bja/aet406 PMid:24318857

23. World Health Organization. Minimal Information Model for Patient Safety Incident Reporting and Learning Systems. Geneva: World Health Organization; 2016.

24. Farley DO, Damberg CL. Evaluation of the AHRQ Patient Safety Initiative: Synthesis of Findings. 2008;44(2 Pt 2):756-76.

PMid:21456115

25. Minnesota Department of Health. Adverse Health Events in Minnesota. in Adverse Health Events in Minnesota. Minnesota: Minnesota Department of Health; 2018. p. 141-60. https://doi org/10.4324/9781315167596-10

26. Elliott $P$, Martin $D$, Neville D. Electronic clinical safety reporting system: A benefits evaluation. JMIR Med Inform. 2014;2(1):e12. https://doi.org/10.2196/medinform.3316 PMid:25600569

27. Hwang JI, Lee SI, Park HA. Barriers to the operation of patient safety incident reporting systems in Korean general hospitals. Healthc Inform Res. 2012;18(4):279-86. https://doi.org/10.4258/ hir.2012.18.4.279

PMid:23346479

28. Dekker S. Patient Safety a Human Factors Approach. London: CRC Press; 2011.

29. Cima RR, Lackore KA, Nehring SA, Cassivi SD, Donohue JH Deschamps C, et al. How best to measure surgical quality? comparison of the agency for healthcare research and quality patient safety indicators (AHRQ-PSI) and the American college of surgeons national surgical quality improvement program (ACS-NSQIP) postoperative adverse events at a single institution. Surgery. 2011;150(5):943-9. https://doi.org/10.1016/j. surg.2011.06.020

PMid:21875734

30. Harkanen M, Saano S, Vehvilainen-Julkunen K. Using incident reports to inform the prevention of medication administration errors. J Clin Nurs. 2017;26(21-22):3486-99. https://doi. org/10.1111/jocn.13713

PMid:28042673

31. Derosier J, Stalhandske E, Bagian JP, Nudell T. Using health care failure mode and effect analysis ${ }^{\mathrm{TM}}$ : The VA national center for patient safety's prospective risk analysis system. $J$ Qual Improv. 2002;28(5):248-67, 209. https://doi.org/10.1016/ s1070-3241(02)28025-6

PMid:12053459 